\title{
DEL ÁREA A LA INTEGRAL. UN ESTUDIO EN EL CONTEXTO EDUCATIVO
}

\author{
TURÉGANO MORATALLA, P. \\ Departamento de Matemáticas. Universidad de Castilla-La Mancha.
}

\section{SUMMARY}

We present in this paper the development and results of some research into the concepts of definite integral which manifest themselves in students when this concept is introduced to them through its geometric definition and independently of the derivative.

\section{EL PROBLEMA DE INVESTIGACIÓN. PLANTEAMIENTO Y DESARROLLO DEL TRABAJO}

Recogemos en este informe un resumen de tesis doctoral (Turégano, 1994) realizada sobre un tema del currículo de bachillerato en España y en otros países: la integral definida. Podemos enmarcarlo dentro de los temas que integran lo que llamamos «pensamiento matemático avanzado». Los conceptos de las matemáticas avanzadas tienen una complejidad intrínseca, pues cada uno de ellos se basa en conceptos más elementales y no puede entenderse sin una sólida $\mathrm{y}$, a veces, muy específica comprensión de éstos.

El trabajo tiene su origen en la reflexión que la investigadora, en un momento determinado de su práctica educativa, hace acerca del fracaso de sus alumnos en la comprensión de los conceptos de cálculo, en general, y de la integral definida, en particular. Esta reflexión le lleva a darse cuenta de que, hasta que no tienen 16 años aproximadamente, los estudiantes no oyen hablar del infinito, de la idea de límite, etc. La enseñanza del cálculo no pasa por una fase previa de carácter experi- mental. El resultado es que, a partir de ese momento, los estudiantes deben asimilar al mismo tiempo los fenómenos asociados a las apariciones del infinito y de los límites, y los conceptos y teorías formales que los expresan y desarrollan matemáticamente. Interviene ahí una teoría que no tiene como función ordenar un conjunto rico en experiencias previas, simplemente porque tal conjunto no existe. Freudenthal (1973) ha puesto en evidencia las dificultades que resultan de este estado de cosas.

Tras un análisis exhaustivo de la bibliografía sobre las investigaciones realizadas en ese campo, hemos constatado que los estudiantes no tienen un rendimiento aceptable en los cursos de cálculo, ni siquiera en la universidad. Las causas que explican esta realidad las encontramos en el terreno epistemológico (Sierpinska, 1985a y b, 1987a y b, 1989), en el terreno didáctico (Orton, 1979, 1983a, 1983b) y en el terreno psicológico (Tall y Vinner, 1981; Vinner, 1991). 
En cuanto a nuestro objeto de investigación, la integral definida, existen estudios cuyas conclusiones hablan por sí mismas de la necesidad de un cambio de enfoque conceptual:

- El punto de vista que, a menudo, es «impuesto» al estudiante de instituto y de universidad es el de la integral de Riemann, en la que el área ya no es definida como un objeto geométrico, sino como el resultado de un cálculo según un procedimiento dado. ¿Por qué no pensamos en la dificultad que puede suponer para el estudiante el relacionar el área con el proceso de sumación que permite sumar infinitas cantidades «infinitamente pequeñas»? Y, aunque sea una forma de razonar muy sugestiva y útil, frecuentemente, desde el punto de vista lógico, adolece del defecto de no poder atribuir un significado exacto al concepto de cantidad infinitamente pequeña (Schneider-Gilot, 1988). ¿Por qué no pensamos también en la dificultad de las tres magnitudes que hay presentes cuando definimos la integral de Riemann: los rectángulos, los segmentos a los que se reducen y el área curvilínea que se pretende determinar?

- Por otra parte, «parece que referirse a la integral como límite de una suma no responde a la lógica de los infinitésimos» (Cordero, 1987).

- Orton (1983a), en su estudio de la comprensión que tiene el estudiante de la integración, concluye que los cuatro ítems que trataban sobre la comprensión de la integración como límite de una suma constituyen la dificultad principal.

- La aproximación mediante sumas «superiores»e «inferiores», por ejemplo, de rectángulos (integral de Riemann) se construye sobre un teorema de existencia que asegura que esas sumas existen sin llegar nunca a justificar al estudiante novel, ni su existencia, ni su convergencia (Tall, 1986).

A la luz de estos resultados, consideramos que la investigación debía trascender, en primer lugar, a los contenidos matemáticos mismos, y, en este caso concreto, a la reconstrucción del currículo vigente, y, en segundo lugar, al modo de transmisión de esos contenidos.

A ello se debe que encontremos en ella dos fases muy diferenciadas:

- una primera fase en el campo del desarrollo de las ideas matemáticas, $\mathrm{y}$

- una segunda fase que tiene lugar en el campo de la investigación educativa.

Como es natural, cada una de ellas se afronta con objetivos distintos y, por ello, con distintos medios para su consecución.

La finalidad de la primera fase fue elaborar un modelo, dentro del contexto matemático, que pudiera utilizarse para diseñar una propuesta didáctica que permita presentar el concepto de integral definida a los estudiantes que van a ser iniciados en el estudio del cálculo infinitesimal.

La segunda fase tiene lugar en el campo de la investigación educativa, y la llevamos a término en cuatro etapas, de las que haremos referencia más adelante, cuya finalidad es estudiar las imágenes del concepto de integral definida que se ponen de manifiesto en los estudiantes después de una etapa de aprendizaje.

Nuestra hipótesis de trabajo es que los estudiantes pueden aprender (de forma intuitiva) conceptos de cálculo sin el dominio previo o simultáneo de las usuales habilidades algorítmicas, utilizando la visualización a través del ordenador para dar significado al concepto de integral definida y a sus propiedades mediante la idea de área bajo una curva.

\section{MARCO TEÓRICO DE LA INVESTIGACIÓN}

El conocer el desarrollo histórico de un concepto nos puede ayudar a comprender cómo se produce el proceso de aprendizaje de ese concepto - por qué aparecen determinadas dificultades o errores- o a organizar su enseñanza.

Esta metodología de investigación se basa en una hipótesis según la cual los problemas que ha tenido que resolver la humanidad para llegar al conocimiento que tenemos hoy de un concepto determinado son paralelos a los problemas que tiene que superar un estudiante actual para comprender correctamente ese concepto. Se trata, por tanto, de emparejar el proceso de aprendizaje con ese desarrollo histórico, evitando el error de creer que los estudiantes recorren los mismos caminos por los que se ha desarrollado la historia o que hay que conducirlos paso a paso, pues las condiciones actuales son muy diferentes de las anteriores.

Si en la primera fase pretendíamos estudiar el origen y evolución del concepto de integral definida a lo largo de la historia y cómo ha sido transmitido, y en la segunda queríamos estudiar las «imágenes del concepto» ${ }^{1}$ que se crean en los estudiantes y su evolución en el sentido psicológico, era lógico pensar en un marco epistemológico y en otro psicológico, y que ambos marcos se pudieran unir de forma coherente.

Estudiamos cómo Lakatos (1976) reconstruye la evolución histórica del conocimiento matemático ${ }^{2}$ y el análisis que hacen Tall y Vinner (1981) y Vinner (1991) acerca de cómo se crean las imágenes del concepto y su evolución en la mente de los estudiantes. Tratamos de establecer un paralelismo entre los términos empleados por Lakatos y Vinner, y asumir, como marco epistemológico, el primero y, como marco psicológico, el segundo.

Así, la familiaridad con el concepto de la que habla Lakatos se puede identificar con la imagen del concepto de Vinner. Los monstruos pueden verse como los atribu- 
tos, propiedades, etc., que no están incluidos en la imagen del concepto. El largo proceso de pruebas y refutaciones, lo asociamos con el estudio de ejemplos, contraejemplos y análisis de propiedades. La exclusión de monstruos supondría una expansión de la imagen del concepto. Por último, cuando Lakatos habla del concepto generado por la prueba, podría verse como la imagen del concepto generada por la experiencia del estudiante.

Se ha tenido en consideración el papel que juegan en la formación de la imagen del concepto: los procesos de resolución de problemas y realización de tareas, los errores, los procesos visuales, la utilización del ordenador y las distintas secuenciaciones del currículo.

\section{PRIMERA FASE DE LA INVESTIGACIÓN}

La preocupación por entender más profundamente las raíces de los conceptos fundamentales de la matemática -en concreto, el concepto de área y su relación con la integral-y así responder mejor a los problemas planteados por su aprendizaje es lo que nos empujó a estudiar el tránsito natural noción-concepto-definición para una de las ideas centrales de la matemática: el área de una región. La finalidad ha sido precisar su desarrollo histórico en cuanto a creación ${ }^{3}$ y transmisión ${ }^{4}$ del conocimiento se refiere, para extraer de ahí pistas de reconstrucción del saber como objeto de comunicación en los procesos educativos actuales (Filloy, 1981). Los medios generales utilizados para la consecución del primer objetivo han sido el estudio de fuentes primarias y de historiadores, el análisis de manuales, tratados y textos, y el estudio de currículos.

Entre las observaciones más relevantes acerca de la génesis del concepto, destacamos:

- Históricamente, a la relación numérica relativamente sofisticada de área le precede la comparación de áreas. Ya en el papiro Rhind, copiado por Ahmes (aproximadamente $1650 \mathrm{aC}$ ), se pone en evidencia un comienzo de teoría de congruencia.

- La clave de la comparación de figuras curvilíneas y rectilíneas, la daría Eudoxo (408-355aC): inscribir y circunscribir figuras rectilíneas a la figura curvilínea y proceder a multiplicar el número de caras o lados indefinidamente, con lo que las figuras rectilíneas se iban aproximando cada vez más a la curvilínea. Lo que no sabían era cómo cerrar el razonamiento, ya que la idea de límite les era desconocida.

- Se han identificado tres definiciones diferentes del concepto de integral a lo largo de la historia:

- Antes del siglo XVIII encontramos numerosos ejemplos de cálculos de áreas bajo curvas, que fueron realizados con independencia del trazado de tangentes. Éste es el primer concepto de integral definida: «cuadratura con independencia de tangentes».
- A partir de la obra de Euler (1707-1783), aparecen las funciones en lugar de las curvas como objeto de estudio, lo que permitió la gradual aritmetización del análisis y su consecuente separación de la geometría. El problema de la integración era el de determinar una función primitiva $F(x)$ que tuviera como derivada $f(x)$. Durante el siglo XVIII y parte del XIX, el cálculo integral es definido como el inverso del diferencial.

- Durante el siglo XIX se produce un cambio conceptual de lo que es una función: se acepta la definición de función de una variable real como correspondencia arbitraria entre números reales.

Esta nueva situación lleva al planteamiento siguiente: Si se pueden considerar funciones arbitrarias, y éstas no se pueden expresar como ecuaciones, ¿tiene sentido preguntarse por el significado de la integral sobre estas funciones? Inevitablemente, tiene que surgir un nuevo concepto de integral. Cauchy (1789-1857) presenta una definición de integral «como límite de una suma», y una formulación rigurosa del teorema fundamental del cálculo. La definición de integral como límite de una suma nace en un contexto de fundamentación teórica, pero, una vez definida, se prescinde de ella para abordar las aplicaciones. La necesidad del tratamiento de CauchyRiemann no está en el cálculo de áreas y volúmenes, ni tampoco en dar una definición de área.

- Peano, en 1887, da la primera definición formal de área. Retoma, como punto de partida para su definición, la idea de Eudoxo y su método de exhausción.

Finalmente, Lebesgue, en los primeros años del siglo $\mathrm{XX}$, precisa las indicaciones de Borel sobre la definición de Peano-Jordan y expone su teoría de la medida, que ha sido muy fecunda y ha servido de base para la teoría más general de integración. Regresamos a los métodos intuitivos anteriores a Cauchy, pero la definición de medida les da una fundamentación lógica sólida.

En el detallado estudio de los cuestionarios oficiales y de los textos de los siglos XIX y XX en nuestro país, observamos que, hasta la implantación en 1990 de la Ley Orgánica General del Sistema Educativo (LOGSE), el tratamiento dado a los conceptos de área y de integral no favorecía el poder establecer una adecuada conexión entre ellos, fundamentalmente porque no se tenían en cuenta simultáneamente las tres etapas que consideramos clave en el aprendizaje:

- construir la noción de área como magnitud autónoma;

- construir una aplicación «medida» entre superficies y números que se pueda extender al máximo de superficies planas;

- construir el concepto de integral partiendo del concepto de área.

Con la entrada en vigor de la LOGSE, esta secuencia, en el aspecto curricular, ya es posible, pues se explicita claramente que se ha de construir la magnitud antes de definir la aplicación «medida» que permite asignarle un 
número. En lo que respecta al cálculo infinitesimal, se recomienda empezar por un tratamiento intuitivo de las funciones, a la vez gráfico y analítico, e introducir el concepto de integral definida a partir del cálculo del área definida bajo una curva. Bien es cierto que se hace alusión al método genético, pero éste no queda reflejado en la propuesta de actividades ni de contenidos, ya que la organización de estos últimos sigue siendo la secuencia lógica del desarrollo del cálculo y no la genética.

Según estos resultados, pensamos que, para iniciar al estudiante en el estudio del cálculo infinitesimal, sería más adecuado utilizar una secuenciación del currículo del cálculo y un enfoque de la integración, de acuerdo ambos con la génesis histórica. Proponemos comenzar con la integral definida, independientemente de la diferenciación y como primera introducción al concepto de límite; todo ello motivado por el problema que está en el origen del cálculo integral: el cálculo de áreas planas. Los argumentos que esgrimimos para ello son los siguientes:

- Como principio general, es probable que, cuando una cosa se descubre antes que otra, la primera es más sencilla que la segunda. Más de mil años antes de que nadie conociera los métodos de la diferenciación, Arquímedes ya está haciendo integrales definidas.

- La integral es una continuación de la idea de área, que los estudiantes conocen desde los primeros días de la escuela, y, como decía Lebesgue: ¿No entenderían los estudiantes más fácilmente que, al pasar de la geometría al análisis, nada ha cambiado sino el lenguaje, que era más geométrico antes, pero más analítico después?

- El estudiante llega a convencerse realmente de que hay un número que mide el área, hecho que en nuestra propuesta es intuitivamente obvio. La idea de límite, necesaria para hallar la pendiente de una tangente a partir de la pendiente de una cuerda, es conceptualmente más difícil.

- El argumento matemático más importante para hacerlo así es que se evita a los estudiantes el considerar la integración principalmente como la operación inversa de la diferenciación. Los estudiantes pueden ver entonces el teorema fundamental del cálculo, que une la integración con la diferenciación, como lo que realmente es: un robusto puente que une estas dos estructuras matemáticas aparentemente independientes (Turégano, 1994, p. 128).

\section{Modelo teórico para la construcción conceptual de la integral definida}

El modelo que proponemos para introducir conceptualmente la integral en principio definida -y que consideramos una clara alternativa a la integral de Riemann utilizada habitualmente en la enseñanza- está basado en la definición geométrica de integral presentada por Lebesgue (1928). Goza, por tanto, de la notable ventaja de la integral de Lebesgue con respecto a la de Riemann ${ }^{5}$.

Lo que hemos hecho ha sido traducirlo a un modelo asequible a los estudiantes de bachillerato para que puedan entender que la noción de integral va asociada a la idea de medir una región, y les hemos proporcionado los medios para que la puedan medir sin recurrir al cálculo de primitivas. En definitiva, hemos retomado la primera imagen del concepto de integral, con la enorme ventaja de que hoy disponemos del ordenador para visualizar y realizar los tediosos pero necesarios cálculos.

La idea es presentar la integral como una continuación de la noción de área, que los estudiantes conocen desde los primeros días de la escuela. Lo que empezó para los estudiantes de educación primaria con la medición de áreas de figuras en general, rectilínea o curvilíneamente limitadas, debe continuar en secundaria con el estudio de clases muy especiales: a saber, aquéllas que están limitadas por una curva sólo por arriba o por abajo. De hecho, estas figuras no son otra cosa que representaciones gráficas de una función $f(x)$ en un intervalo $a \leq x \leq b$. El problema de calcular el área de esas figuras consiste en determinar un dominio ${ }^{6}$ rectangular (Fig. 2) cuya área sea igual a la del dominio original (Fig.1), quedando el problema reducido al cálculo de la altura media de la función $f(x)$ en todo el intervalo $[a, b], y$, a continuación, calcular el área pedida como el producto de esta altura por la longitud $b-a$ del intervalo cerrado $[a, b]$.

Se ha utilizado desde el principio el símbolo usual de integración, presentándolo como una forma de representar algo que ya nos es conocido: el área de una región. La razón para utilizar el símbolo abreviado

$$
\int_{a}^{b} f(x)
$$

es que expresa, con más fuerza, que la integral depende sólo de la función $f$ y del intervalo $[a, b]$.

Figura 1

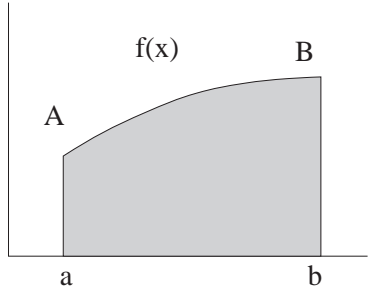

Área $a b B A=$ Área $a b C D$

$$
\int_{a}^{b} f=(b-a) \cdot M
$$

En el caso de que $f(x)$ no sea siempre positiva, la curva $A B$ corta el eje $x$ en un número finito o infinito de veces. En este caso se tienen dos clases de dominios: unos, por debajo de $O x$; los otros, por encima. Cada uno de estos dominios es cuadrable (Lebesgue, 1928, p. 41), y se define la integral de $f(x)$ como la suma de las áreas que 
están por encima del eje $o x$ menos la suma de las áreas que están por debajo de dicho eje.

Comencemos con una función real $f(x)$ definida en un intervalo $[a, b]$, acotada y no negativa. El problema, tal como lo hemos planteado, se reduce a calcular la altura media. Realicemos biparticiones consecutivas del intervalo $[a, b]$ en $2,4,8, \ldots, 2^{n}$ partes iguales, de tal forma que a cada entero positivo $n$ le asociamos una subdivisión del intervalo en $2^{n}$ partes iguales que llamaremos $n$-ésima subdivisión.

Consideremos, a continuación, una $n$-ésima subdivisión y denotemos con $h_{i}$ y $H_{i}$, respectivamente, el ínfimo y el supremo de la función $f(x)$ en el $i$-ésimo intervalo de la subdivisión. Es evidente que estos números, $h$ y $H$ están bien definidos por la hipótesis de que $f(x)$ es acotada, y, a partir de ellos, podemos definir los números $h(f, a, b, n)$ y $H(f, a, b, n)$ por las relaciones siguientes:

$$
\begin{aligned}
& h(f, a, b, n)=\frac{1}{2^{n}} \sum_{i=1}^{2^{n}} h_{i} \\
& H(f, a, b, n)=\frac{1}{2^{n}} \sum_{i=1}^{2^{n}} H_{i}
\end{aligned}
$$

Estos números representan las alturas medias mínimas y máximas en relación con la $n$-ésima subdivisión. De su definición se deducen, para todo entero positivo $n$, las relaciones siguientes:

$$
\begin{aligned}
& \text { 1. } h(f, a, b, n) \leq h(f, a, b, n,+1) \\
& \text { 2. } \mathrm{H}(f, a, b, n \geq H(f, a, b, n,+1) \\
& \text { 3. } h(f, a, b, n) \leq H(f, a, b, n)
\end{aligned}
$$

Estas relaciones nos indican que las sucesiones $\{h(f, a, b, n)\}$ $y\{H(f, a, b, n)\}$ para $n=1,2,3, \ldots$ son monótonas y acotadas, por lo que ambas tienen límite cuando $n \rightarrow \infty$ :

$\lim _{n \rightarrow \infty} h(f, a, b, n)=\sup _{n} h(f, a, b, n)=h(f, a, b)$

$\lim _{\mathrm{n} \rightarrow \infty} H(f, a, b, n)=\inf _{n} H(f, a, b, n)=H(f, a, b)$

con $h(f, a, b, n) \leq H(f, a, b, n)$.

Los números hallados, que identificamos con la altura media mínima y máxima, respectivamente, no se puede asegurar que sean iguales, pero, en caso de serlo, ese número que denotaremos por $M(f, a, b)$ es la altura media de la función $f(x)$ en el intervalo $[a, b]$.

Definimos la integral ${ }^{7}$ de la función $f(x)$ en el intervalo $[a, b]$ como el producto de $b-a$ por el número $M(f, a, b)$ :

$$
\int_{a}^{b} f(x) M(f, a, b,)(b-a)
$$

Corolarios. La definición dada es independiente del signo de la función $f(x)$ e, incluso, del signo de $b-a$, de manera que se verifica:

$$
\begin{aligned}
& \int_{a}^{b}(-f)=-M(f, a, b)(b-a)=-\int_{a}^{b} f \\
& \int_{a}^{b}-f=M(f, a, b)(b-a)=-M(f, a, b,)(a-b)=-\int_{a}^{b} f
\end{aligned}
$$

\section{SEGUNDA FASE DE LA INVESTIGACIÓN: PARTE EXPERIMENTAL}

La segunda fase, que ya hemos dicho que tiene lugar en el campo de la investigación educativa, la llevamos a término en tres etapas para poner en práctica nuestra propuesta y una cuarta etapa para entrevistar a los estudiantes después del aprendizaje.

\section{Etapa 1}

La muestra fue tomada al comienzo del curso 1991-92. Inicialmente, estaba compuesta por 87 estudiantes pertenecientes a tres grupos naturales de primer curso de BUP (14-16 años) de tres institutos de Albacete.

Los objetivos propuestos en esta etapa fueron los siguientes:

- Describir la situación de los estudiantes de la muestra en cuanto a su rendimiento en cuestiones relacionadas con el infinito, visualizaciones y áreas.

- Delimitar perfiles o clases de estudiantes basándose en las respuestas y estrategias de resolución utilizadas por ellos en los cuestionarios anteriores y durante la entrevista clínica.

Comenzamos con un diagnóstico sobre la situación de partida de los estudiantes con cuestiones relacionadas con la intuición que tienen del infinito y su capacidad para visualizar y resolver problemas de áreas, temas que consideramos que pueden influir en la formación de las imágenes del concepto de integral. Se les administraron tres cuestionarios, en días diferentes, dentro de su horario habitual de clase por su profesor de matemáticas.

De los datos obtenidos a partir de la aplicación de los cuestionarios, elegimos el análisis factorial de correspondencias como método estadístico para clasificar a los estudiantes, y, a partir de esta clasificación, determinamos la muestra para las entrevistas (27 estudiantes: 9 de cada curso). Para la aplicación de este método empleamos el programa CHADIC.vs versión 3.0 del Départament Informatique de l'IUT de Niza.

Esta muestra nos va a permitir, por medio de entrevistas, reclasificar a los estudiantes, no sólo de acuerdo con su rendimiento sino también de acuerdo con sus estrategias (Turégano, 1994).

De las muchas conclusiones obtenidas, destacaríamos, en relación con el área, las siguientes:

- Cuando se trata de reconocer la igualdad de áreas, la congruencia entre regiones diferentes por medio de 
equidescomposición y equicomplementación, o a través de otra magnitud, se revela como el método mejor aceptado por los estudiantes. Estos métodos llevan implícito el reconocimiento de la conservación del área y de la propiedad aditiva.

- Los cortes laminares por planos paralelos o descomposiciones infinitesimales de las superficies no son procedimientos evocados espontáneamente para el cálculo de áreas por los estudiantes, ni en el caso de figuras rectilíneamente limitadas ni curvilíneas. Quizá esto se debe a tener que romper con la imagen que tienen del área como espacio que se cubre.

- La imposibilidad de «ver» un área como límite de una suma de infinitesimales tiene su origen en que «pasan» al indivisible de una dimensión menor antes de sumar las áreas, y en el paso al límite les «desaparece» el área. Esto pone en evidencia que el cálculo de un área bajo una curva definida por una función crearía problemas al tratar de remitir a los indivisibles de longitud $y=f(x)$.

- Se observa en los estudiantes, tanto en el cuestionario de «infinito» como en el de «áreas», un empleo tácito del infinitesimal como algo muy pequeño pero distinto de cero, nunca como variables que convergen en cero.

La primera de estas conclusiones apoya nuestra propuesta en el sentido de que los estudiantes admitirían la congruencia del área bajo una curva con la de un rectángulo, y las otras tres ponen de manifiesto las dificultades que encontrarían los estudiantes con la definición de integral de Riemann.

Con respecto al concepto de infinito, la concepción que tienen los estudiantes del infinito potencial es el mayor obstáculo para concebir un proceso infinito como algo definido o acabado. Ésta es una de las razones por las que el proceso de integración hay que terminarlo; no nos podemos quedar en aproximaciones al área. No se puede dejar al estudiante ante la eterna duda de si el límite se alcanza o no se alcanza, pues estaríamos afianzando más su concepción del infinito potencial. Hay que plantearle situaciones que le hagan romper con esta imagen.

En el campo de la visualización, hemos determinado que los estudiantes remiten con mucha más frecuencia a un análisis parcial de las figuras (que les permite pasar de las partes al todo) que a un análisis global. Aquí también tendrían problemas con la integral de Riemann.

El enfoque que hemos planteado para la integral está en consonancia con las ideas previas de los estudiantes acerca del concepto de área y con la génesis del concepto de integral. Ahora nos planteamos cómo debe ser la transmisión de estos contenidos, y ahí es donde entran en juego la utilización de las nuevas tecnologías y los resultados de las investigaciones acerca de cómo se crean y evolucionan las imágenes del concepto en la mente de los estudiantes.

Todas estas consideraciones se plasman de forma explí-

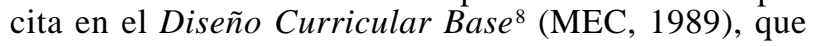

recoge las bases de la propuesta oficial española para la educación primaria y secundaria en la actualidad.

\section{Etapa 2}

Con el modelo teórico elaborado en la primera fase de la investigación, y de acuerdo con los conocimientos previos e «intuiciones» ${ }^{9}$ manifestadas por los estudiantes en las entrevistas, diseñamos las «situaciones didácticas» ${ }^{10}$ sobre la base del «modelo constructivista» ${ }^{11}$ del aprendizaje. Conocer las ideas previas de los estudiantes nos permite actuar sobre la imagen del concepto para transformarlas o mejorarlas. Tall (1985) recomienda hacerlo con organizadores genéricos, que son micromundos (por ejemplo, programas de ordenador, materiales curriculares, etc.) dentro de los cuales el estudiante puede manipular un concepto u objetos relacionados con él.

Hemos elaborado nuestros propios programas de ordenador con la finalidad de proporcionar un contexto rico en que se pueda explorar el proceso de construcción del concepto y discutir el propio concepto, permitiendo al estudiante crear su «imagen del concepto» de integral. No hay duda de que existe un creciente cuerpo de investigación (Tall, 1986; Thomas, 1988; Heid, 1988; Turégano, 1994) que aboga por el uso del ordenador en los cursos de cálculo invitando a un cambio curricular centrado en los conceptos de tal forma que nos permita abordarlos con varias representaciones y nos posibilite conexiones entre ellas.

\section{Etapa 3}

Las conclusiones obtenidas en la primera etapa nos permiten, dado que los estudiantes presentan éxitos y fracasos similares en los tres institutos, reducir la muestra a un solo instituto (instituto núm. 3) para afrontar la etapa de aprendizaje con los objetivos siguientes:

- Observar las respuestas espontáneas y debates de los estudiantes en el aula cuando, con la ayuda del ordenador, resuelven por parejas problemas de áreas y exploran el concepto de integral definida. (Los medios específicos utilizados son los problemas propuestos, programas de ordenador que les facilitamos y la metodología de trabajo.)

- Describir, en momentos determinados del aprendizaje, el rendimiento de los estudiantes en cuestiones relacionadas con conceptos y destrezas necesarias para la comprensión del concepto de integral. (Los medios utilizados fueron pruebas de lápiz y papel, presentando los resultados bajo criterios cuantitativos y análisis de errores cometidos.)

La etapa de aprendizaje se llevó a cabo en ocho sesiones en el mes de mayo de 1992 (Turégano, 1994).

Partimos de que el estudiante está familiarizado con el concepto de área como magnitud y tiene ciertas intuiciones sobre el infinito y el concepto de límite, si bien tiene una imagen del concepto un poco ingenua. Queremos estudiar si el estudiante es capaz de evolucionar en su 
imagen del concepto mediante su trabajo con el material curricular que se le presenta y con los programas de ordenador utilizados. La situación-problema de la que nos vamos a servir aquí es la de presentar sucesiones que convergen en un mismo número, apoyando esta situación con una imagen visual a través del ordenador que les haga sentir la necesidad de modificar o romper con la imagen de que el límite «no se alcanza». Dado que las ideas sobre los límites empiezan a desarrollarse cuando se pueden explorar las situaciones en las que una sucesión se establece o converge, y que esto puede ser más real en una situación geométrica, nos hemos aprovechado de ello y, con la ayuda del ordenador, los estudiantes visualizan que cierto resultado es plausible, y, de esta manera, pueden entrar en conflicto con sus intuiciones.

Además, las sesiones de aprendizaje tienen la finalidad de que los estudiantes adquieran una familiaridad con el concepto de integral definida, es decir -y utilizando la terminología de Vinner-, adquieren una determinada imagen del concepto.

Se selecciona una colección de ejemplos y contraejemplos, es decir $a$ ) por una parte, funciones en las que, al construir gráficas, el valor numérico de la integral sea exactamente el del dominio sombreado, para lo cual $f(x) \geq 0$ en el intervalo considerado: y $b$ ) por otra parte, funciones que nos determinan dominios sobre el eje $o x$ y debajo de dicho eje en el intervalo considerado, con lo que el valor de la integral será el área de los dominios con $f(x) \geq 0$ menos el área de los dominios con $f(x) \leq 0$, con la finalidad de que construyan una imagen correcta del concepto de integral.

Aquí se requiere una puesta en común con la finalidad de negociar una definición para la integral. Tomamos, por ejemplo, la función $f(x)=x^{3}$ en el intervalo [-2,2]. Si se aplica el programa INTEGRAL, el valor obtenido para el área será 0 , pero, según la imagen mostrada, claramente hay un área. Muchos estudiantes exclaman incrédulos: « No puede ser!» Hay una fe ciega, por parte de los estudiantes, en que el ordenador no se equivoca, por lo cual hay algo más. Aquí hay una oportunidad para la investigación y para llegar a dar una definición geométrica de integral. Es en este momento cuando hay que descomponer la imagen holística del gráfico en partes para proceder a su análisis, no en entidades discretas, aisladas unas de otras, sino relacionadas en un todo. Se analiza, por una parte, el dominio cuya área es $\int_{-2}^{0} x^{3}, \mathrm{y}$, por otro, el dominio de área $\int_{0}^{2} x^{3}$, pero siempre tratando de relacionarlas con $\int_{-2}^{2} x^{3}$.

Merece la pena comentar brevemente la situación con la que nos podemos encontrar en la resolución de este problema, ya que se observa una clara división dicotómica en el pensamiento de los estudiantes:

- Unos las justifican o deducen partiendo de los números que determinan (medida del área).
- Otros las deducen partiendo de la interpretación geométrica (área como magnitud) recurriendo a los rectángulos.

Los primeros justifican su razonamiento de la forma siguiente: al calcular $\int_{-2}^{2} x^{3}=0$, y tener a la vista el gráfico con el área sombreada, deducen que esa área no puede ser cero. Un análisis por partes les permite calcular el área entre $[-2,0]$ y entre $[0,2]$. Al ver que son iguales, pero con signo opuesto, dicen que habrá que restar en vez de sumar.

Los segundos argumentan que, si el área entre $[-2,0]$ es negativa, como esa región es congruente con la de base $[0,2]$, el área sería $\int_{0}^{2} x^{3}-\int_{-2}^{0} x^{3}$. Alguno, incluso, llega a insinuar si el área no será el doble de la de arriba: $\int_{-2}^{2} x^{3}=2 \int_{0}^{2} x^{3}$.

La conclusión a la que llegan es que, cuando la gráfica de una función corta el eje $x$ en varios puntos, determina regiones sobre y bajo dicho eje. El área buscada no es la suma de áreas, ya que, al ser la de abajo negativa, la estaríamos restando, y llegan a la conclusión de que $\int_{a}^{b} f(x)$ no se debe hacer de «una sola vez» en aquellos casos en que $f(x)$ corte el eje $x$ en más de dos puntos, debiendo descomponerse, entonces, en la suma de tantas integrales como regiones distintas determine.

Es indudable que, sin la imagen visual, estos razonamientos no se hacen explícitos en los estudiantes. Quiero reseñar, además, que el ejemplo es muy significativo, ya que, si eligiéramos otro en que la integral primera no fuera cero, quizá no se hubiera suscitado esta discusión, a no ser que existiesen estudiantes con una estimación visual del área muy precisa.

Con este tipo de actividades podemos ayudar al estudiante en el difícil proceso de transición al concepto abstracto, pero ese proceso depende, esencialmente, de las conexiones entre las distintas representaciones que utilicen los estudiantes. Estas conexiones, según Dreyfus (1991a), constituyen una tercera etapa del aprendizaje.

Si damos a los estudiantes la oportunidad de explorar las propiedades de la integral, vamos a ver si han conseguido establecer la conexión entre las representaciones gráfica y numérica de integral, así como su expresión simbólica. Se presentan ejemplos concretos:

- Hallar-si existe- una relación entre $\int_{1}^{3} x^{2}$ y las integrales $\int_{1}^{15} x^{2}$ y $\int_{1.5}^{3} x^{2}$

- Hallar -si existe- una relación entre $\int_{0}^{4}\left(x^{2}+x^{3}\right)$ y las integrales $\int_{0}^{4} x^{2} \mathrm{y} \int_{0}^{4} x^{3}$. 
- Hallar-si existe- una relación entre $\int_{1}^{4} 3 x^{2}$ y la integral $\int_{1}^{4} x^{2}$

De nuevo se observan las mismas tendencias en las respuestas de los estudiantes. Por ejemplo, si nos referimos a la aditividad del integrando y les pedimos que traten de hallar una relación entre $\int_{a}^{b} f, \int_{a}^{b} g$ e $\int_{a}^{b} f+g$, los primeros resuelven las tres y, con los números resultantes de medir el área, escriben la relación $\int_{a}^{b} f+g=$ $\int_{a}^{b} f+\int_{a}^{b} g ;$ los segundos recurren a los rectángulos y escriben la relación entre sus áreas: $(b-a) M_{1}+(b-a) M_{2}$ $=(b-a)\left(M_{1}+M_{2}\right)$, para pasar de ahí a las integrales correspondientes.

Las mismas estrategias de resolución son utilizadas por los estudiantes para todas las propiedades. La ventaja de los que utilizan la interpretación geométrica es obvia, ya que su estrategia les permite realizar generalizaciones; no así a los que utilizan los números, que deben recurrir a ejemplos concretos para llegar a la relación entre las integrales.

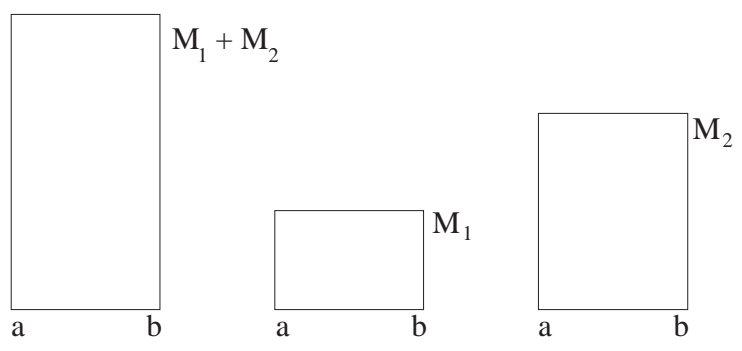

Un grupo pequeño de los que utilizan la imagen geométrica y obtienen bien la relación, posteriormente, validan dicha relación recurriendo a los números, lo que pone de manifiesto la reticencia de algunos estudiantes a dar validez a las demostraciones visuales, tal y como manifiestan Dreyfus y Eisenberg (1990).

De las muchas observaciones que recogimos en esta etapa y que fueron corroboradas en la última entrevista -las cuales obviamente no podemos plasmar aquí por razones de espacio-, destacamos las siguientes:

- Los estudiantes tienen más dificultades en la utilización de la expresión analítica de las funciones que en la visualización de sus gráficos, lo que nos lleva a pensar que tendrán más dificultades en la comprensión del concepto de integral como antiderivada que en el aspecto geométrico de la misma.

- El proceso de integración seguido para determinar el área es apropiado para inducir a los estudiantes a la discusión de determinados conceptos de cálculo -sucesión, límite, número real e integral- en un contexto concreto, que es el del cálculo de áreas bajo curvas, y permite de esta forma organizar la experiencia previa a la formalización de los conceptos de cálculo.

- Los modelos visuales en la enseñanza del cálculo y la eliminación de cálculos algebraicos (mediante el uso del ordenador) favorece la formación y transformación de intuiciones y la creación de imágenes del concepto que ayudarán posteriormente a la formalización de los conceptos de cálculo infinitesimal.

- La clave en la evolución del concepto de límite ha estado en presentarles una situación evidente (altura única en un rectángulo) en un marco geométrico-numérico (en el que hay interacción en todo momento), situación que convence al estudiante de que realmente hay un número que mide la altura. La existencia de la integral queda así justificada con la existencia de la altura.

- El uso del ordenador permite que el estudiante pueda simultanear los aspectos psicológicos que se ponen en juego: abstracción, representación, formación de conceptos, inducción y visualización.

- Una visión holística del gráfico favorece la formación de la imagen de área bajo una curva y la de integral si se procede posteriormente al análisis de sus partes, no como entidades discretas, aisladas unas de otras, sino relacionadas en un todo.

- Observamos - como ya hemos comentado- dos tendencias distintas en los estudiantes al tratar de justificar las propiedades de las integrales: unos eligen los números que determinan la medida del área, y otros, las regiones cuya área hay que determinar. Los primeros deducen de la relación numérica, obteniendo, al resolver las integrales, la relación entre las mismas; los segundos, hallan la relación entre las integrales de la relación entre las regiones cuya área hay que determinar. Los primeros sólo pueden obtener esas relaciones en casos concretos y experimentales; los segundos pueden obtener las relaciones en casos generales.

- Es sabido que, cuando la función que se integra viene definida en dominios divididos, es decir, por diferentes reglas en subdominios diferentes, los estudiantes tienen dificultades para hallar el área bajo la curva, y éstas se ven incrementadas si en uno de esos dominios $f(x)=0$. El acercamiento a la integral que hemos propuesto tiene la ventaja de que, de forma espontánea, los estudiantes utilizan los diferentes dominios para plantear diferentes integrales y asignan área cero cuando $f(x)=0$. Es una transferencia inmediata, una consecuencia natural del enfoque visual de la integral y de la propiedad aditiva del área.

\section{ENTREVISTA POSTAPRENDIZAJE}

Después de la etapa de aprendizaje se realizaron entrevistas a los nueve estudiantes clasificados en la primera etapa como representantes de clase, con la finalidad de 
enfrentarlos con problemas que tienen que ver con el área bajo una curva en tres contextos distintos: matemático, área bajo la curva velocidad y probabilidades geométricas (Anexo). Las tareas presentadas en las entrevistas nos permiten verificar cómo aplican los estudiantes sus imágenes del concepto de integral, cómo reflejan sus errores, cómo modifican, rompen o restringen su imagen del concepto y el propio concepto para poder continuar. Nos permiten, también, verificar cómo realizan la transferencia a contextos distintos de aquél en que se había desarrollado el aprendizaje. Hemos recurrido a los problemas porque, como afirma Dreyfus (1990), el comportamiento matemático se investiga mejor en las situaciones de resolución de problemas.

\section{Imágenes asociadas al concepto de integral definida}

Durante la última entrevista, en la resolución del problema 1, se han identificado tres tipos de imágenes del concepto en los estudiantes con respecto a su acercamiento a la integral definida que hemos designado con los apelativos de primitiva, operativa y descriptiva, con el significado que el diccionario de la Real Academia Española atribuye a estos términos. Las dos primeras palabras ya habían sido utilizadas en un contexto completamente diferente (Azcárate, 1990). Todos los estudiantes ponen en juego la misma imagen del concepto en la resolución de los tres problemas planteados. En Turégano (1994) recogemos los resultados de la última entrevista y la evolución observada en los estudiantes después del aprendizaje acerca del concepto de límite.

- Un estudiante tiene una imagen primitiva del concepto de integral si la asocia con la fórmula $(b-a) \cdot h$, que le permite calcular áreas de figuras «raras».

Al preguntarles por qué la fórmula $(b-a) \cdot h$ no les recuerda la del cálculo del área del rectángulo, dicen lo siguiente:

C66 -No. Ésta es para las figuras «raras». La del rectángulo es base por altura.

C86 -La del rectángulo es base por altura. Sólo hay dos números, y aquí, tres.

E. -Bien, pero con los números $b$ y $a$ determinamos la longitud de la base del rectángulo.

C86 -No sé...

Esta imagen del concepto corresponde a los estudiantes que tenían una imagen primitiva del área, y éste es el mayor obstáculo para poder construir una imagen del concepto de integral. No han construido una imagen específica de integral ni tampoco de área bajo una curva.

No han asimilado ni los conceptos ni los procedimientos estudiados durante la fase de aprendizaje. A pesar de ello, con la ayuda del ordenador y la fórmula $(b-a) \cdot h$, pueden calcular el área bajo la curva con $f(x) \geq 0$. Pero esto, obviamente, no lo transfieren a otros contextos, sino que ejecutan ciertos tipos de ejercicios de forma rutinaria y sin conocimientos específicos de lo que están haciendo. Utilizan el concepto de forma instrumental.

Se observa en estos estudiantes una tendencia generalizada a explicar el significado de cualquier concepto, estrategia, etc., en términos muy simples y escuetos, utilizando casi siempre un lenguaje gestual, y nunca dando una respuesta que contemple más de un aspecto.

- Una imagen operativa del concepto de integral corresponde a aquellos estudiantes que han construido una imagen incompleta de dicho concepto que no les va a permitir resolver correctamente las situaciones cuando $f(x)<0$, pues otorgan el mismo signo a las áreas de dominios situadas sobre y bajo el eje $o x$. Están anclados en el contexto geométrico de las formas, sin dar definitivamente el salto a las funciones. A pesar de ello, al preguntarles si existen las áreas negativas, argumentan lo siguiente:

C74 - Cuando la $h$ es negativa el área es negativa.

C65 - Cuando el gráfico está por debajo del eje, el área es negativa.

E. -¿Por qué?

C65 - Porque la altura es negativa.

Pero esto no lo tienen en consideración a la hora de los cálculos, donde prima la imagen visual de los dominios y la aditividad del área se pone en juego para sumar las áreas en vez de restarlas. Efectúan el paso al límite solamente en su percepción visual, donde las alturas van acercándose hasta converger en un segmento único, lo cual nos vuelve a indicar que todavía no hay una interacción entre el marco visual y el numérico, que permite el cálculo de la altura media. Ahora bien, son conscientes de la necesidad de alcanzar el límite para determinar realmente el área pedida. Así, por ejemplo:

E. $-¿$ Crees que en cualquier momento $H$ - $h$ será cero?

C74 [Saca un listado con 12 biparticiones.] - Ya es casi cero: $H-h=0$ '00277.

E. $-¿$ Realmente llega a ser cero?

C74 ... Sí, porque en el ordenador se ve que se monta una encima de la otra, y el rectángulo sólo puede tener una altura, y rectángulo sólo hay uno.

E. -¿Qué quieres decir con que sólo hay un rectángulo?

C74 -Bueno, hay muchos, pero como tiene la base fija, sólo hay uno con esa base de la misma área.

E. - ¿Por qué crees que buscamos una altura media?

C74 -Porque, si calculamos el área cuando las $h$ y $H$ aún no coinciden, tenemos dos rectángulos: uno de área mayor y otro de menor que la que queremos calcular.

Justifican la existencia del límite basándose en la unicidad del área a través de la unicidad de la altura única para el rectángulo. La expresión verbal es imprecisa, aunque utilizan correctamente la forma simbólica y figural, escribiendo bien el signo de integración y sus extremos, y sombreando correctamente el área bajo la curva. Éstos son estudiantes que entienden los subconceptos y domi- 
nan destrezas necesarias para la comprensión total del concepto, pero no los han integrado para formar un «todo» coherente en un solo concepto -el de integral definida-, fundamentalmente porque no han dado el salto de la geometría al análisis. Justifican y utilizan bien las propiedades de la integral siempre que $f(x) \geq 0$, y pueden transferir a otros contextos, pues han entendido la relación integral-medida aunque no la calculen bien.

- Una imagen descriptiva del concepto de integral corresponde a aquellos estudiantes que pueden dar una descripción de integral definida con una expresión verbal precisa en lo referente a sus partes y propiedades, y que articulan correctamente los tres tipos de información -figural, simbólica y semántica-, consiguiendo integrar en el concepto todos los subconceptos subyacentes y los procedimientos de cálculo.

En ellos se mezcla una visión geométrica con la numérica, efectuando el paso al límite, tanto en forma de percepción visual, donde las alturas van acercándose hasta converger en un segmento único, como en el aspecto numérico, donde la sucesión diferencia de las alturas máximas y mínimas converge en cero.

E. $-¿$ Crees que en algún momento $H-h$ será cero?

C69-Sí, porque si hacemos más biparticiones, cada vez es la mitad del que tienes [se refiere a la formación del término siguiente], y así cada vez.

E. -Muéstrame por qué.

C69 Con 8 biparticiones, $H-h=0$ '00926; con 9, será 0’00926 / 2 = 0'00463, y con... 1000, es pequeñísimo, y con infinito, cero.

$$
\cdots
$$

E. -Podrías decirme cuánto valdrá la altura del rectángulo?

C69 ... Casi seguro -0 ' $66 \ldots$ se ve que $H$ y $h$ van a -0 ' $66 .$. que es cuando $H-h=0$.

$$
\text { ... }
$$

E. - ¿Por qué crees que buscamos una altura media?

C69 Porque si calculo el área del rectángulo con $h$, me da una más pequeña que la que quiero hallar y, si lo hago con $H$, me da mayor. Entonces, cuando $h=H$, ya calculo la que tengo que hallar.

En la entrevista se pone de manifiesto la influencia del aprendizaje en su evolución de la imagen del concepto de limite, ya que se encontraba en una etapa de transición, y su línea de razonamiento resulta particularmente interesante, ya que acepta la existencia de la altura media, no de una forma perceptiva sino aceptando un proceso infinito como algo definido o acabado.

La clave de la evolución en el concepto de límite, pensamos que ha estado en el estudio de la sucesión $\{H(f, a, b, n)-h(f, a, b, n)\}$, ya que el estudiante intuye fácilmente su convergencia en cero, para concluir que $H=h$. En esta situación, las pruebas de naturaleza heurística pueden ser tan fuertes que produzcan convicción, incluso sin demostración rigurosa.
Es más fácil para un estudiante ver que llega un momento en que la diferencia de estas dos sucesiones es cero que razonar independientemente en cada una de ellas como proceso infinito que nos lleva a tal o cual número, ya que esta última idea puede conectar más directamente con las intuiciones del infinito potencial que se puso de manifiesto en las primeras entrevistas y puede continuar siendo un obstáculo para la aceptación del límite como número alcanzable.

Estos estudiantes transfieren bien a otros contextos, lo que pone en evidencia el alto nivel de comprensión del concepto. Sus imágenes del concepto forman un todo coherente y útil para resolver todo tipo de problema que tenga que ver con el concepto de integral en estos niveles de enseñanza. Utilizan correctamente las propiedades de la integral sin tener que recurrir a los números que miden las áreas.

Esta imagen corresponde al aspecto menos intuitivo y más abstracto, y permite al estudiante comprender y explicar situaciones de cálculo de áreas bajo curvas en el sentido de tener una descripción verbal, una imagen gráfica, una expresión simbólica y unos recursos para calcularla.

Para poder tener una imagen descriptiva del concepto de integral es necesario que el estudiante haya desarrollado el concepto de área como magnitud, que admita la divisibilidad infinita de un segmento y la existencia del límite, y que pueda tener una visión holística del gráfico, que, en determinadas ocasiones, puede descomponer en partes para poderlas después integrar de nuevo en un «todo».

Finalmente, y como muestra del papel que juegan los errores en la formación de la imagen del concepto, presentamos el pasaje de una entrevista en que el estudiante cambia los extremos de integración, hecho que fue aprovechado para inducirle a reflexionar sobre la consecuencia que tendría para la integral aceptar como correcto su error.

E. -Tienes que escribir bien los extremos del intervalo: el extremo izquierdo en la parte inferior y el derecho en la superior.

C76 -Es más fácil así, porque yo siempre escribo de arriba a abajo.

E. -Mira, es un convenio: si yo veo la integral escrita como lo haces tú, me puedo equivocar al calcular el área. ¿No lo crees?

C76 ... La altura será igual y la base siempre es $b-a$.

E. $\quad-$ ¡Claro! Si yo pienso que $b$ es el número que hay escrito en la parte superior y $a$ en la inferior, los resto al revés, y ¿qué pasa?

C76 -Que da un número con el signo cambiado.

E. $-i \mathrm{Y}$ ?

C76 -El área te va a dar con otro signo y te puedes confundir. ... Entonces, si escribes en vez de... se cambia el signo, pero el número es igual. 
E. -Si eso ocurre, ¿te convences de por qué no puedes hacerlo?

C76 -Sí, claro.

En este diálogo se corrobora la tesis defendida por Borasi (1987) de que los errores son «trampolines para la indagación» en vez de señales de que algo ha ido mal en el proceso de aprendizaje, $y$, por tanto, son útiles para completar sus imágenes del concepto.

\section{Transferencia a contextos distintos de aquél en que se ha efectuado el aprendizaje}

Con la última entrevista estábamos también interesados en estudiar si este enfoque de la integración permitiría a los estudiantes transferir los conocimientos adquiridos a contextos diferentes del matemático.

Incluimos dos problemas, uno en el que había que reconocer que el espacio recorrido por un coche era el área bajo la curva velocidad y otro de probabilidades geométricas. En estos problemas, una vez identificada su resolución con la de una integral, no presentarían dificultades para los estudiantes con imagen descriptiva u operativa de integral, ya que en los dos casos la gráfica de la función está sobre $o x$.

Problema 2. (Ver Anexo). Cálculo del área bajo la curva velocidad: espacio recorrido

De entrada, suponemos que nada incita a los estudiantes a «ver» el cálculo del espacio recorrido como un área bajo una curva, si no es porque alguno de ellos se para a pensar si este problema tiene algo que ver con los cálculos de áreas bajo curvas que se han estado haciendo en la fase de aprendizaje. Pero nos llevamos una sorpresa.

Hemos observado dos formas distintas de abordar el problema:

- Unos estudiantes que piensan que todo indivisible representa una distancia recorrida durante un tiempo dado, y la suma de los indivisibles representa, pues, la distancia recorrida.

Indudablemente, la idea de que una magnitud se compone de añadidos sucesivos de indivisibles estaría en el origen de esta confusión.

- Otros recurren a la fórmula $e=v \cdot t$, y son los que no presentaron dificultades para «ver» el espacio como un área. Sin lugar a dudas, este enfoque geométrico de la integral favorece enormemente esta asociación.

Las respuestas dadas por los estudiantes se ajustan, pues, a dos modelos de enunciados:

1) El espacio es igual a la velocidad por el tiempo.

Todos los estudiantes presentan una imagen indiscriminada de «velocidad igual a espacio partido por tiempo», comprensible porque es la única que han podido adquirir a través de su aprendizaje.

Tratan de hallar una relación funcional entre las variables que les permita conectar la velocidad y el tiempo con el espacio.

Comentamos a continuación distintos párrafos de las entrevistas. Se les facilita la hoja con el problema, para que lo planteen, en principio, sin resolver. Queríamos comprobar si podían utilizar las propiedades de la integral sin recurrir a la igualdad de los números que miden el área.

E. ¿Cómo calculas la distancia que habrá recorrido a los 20 segundos?

C69 -Ésta es la velocidad [Señala el eje $y$.] y éste el tiempo [Señala el eje $x$.]. Se multiplican y se mide esta área [Señala un rectángulo de base 20 y altura 80.], que es el espacio.

Antes de que pueda responderle se da cuenta de su error.

C69] - ¡No! ¡Este trozo no es! [Se refiere al trozo que queda por encima de la curva.] Es justo el área bajo la curva, porque siempre no va a la misma velocidad.

E. -Bien. ¿Me podrías indicar cómo calcularla?

C69 -Sí. Es la integral entre 0 y 20 de $v(t)$. [Lo escribe.]

E. $-¿$ Qué distancia recorre entre los 20 y 40 segundos?

A la indicación de que lo señale en el gráfico, lo hace correctamente y plantea:

C69 $-\int_{0}^{40} v(t)-\int_{0}^{20} v(t)=\int_{20}^{40} v(t)$.

E. $-¿$ Me puedes explicar por qué lo haces así?

C69 -Así ya resuelvo las dos preguntas.

E. - ¿A qué preguntas te refieres?

[C69] A calcular el espacio entre 20 y 40 y entre 0 y 40 , porque, calculando entre 0 y 40 , como ya conozco el espacio entre 0 y 20 , tengo todas las preguntas resueltas.

Igual razonamiento encontramos en los estudiantes C76, C75 y C82.

2) El espacio es la suma de las «ordenadas» para cada valor $t$.

El estudiante C84 comienza señalando en el gráfico el espacio en $t=20$ segundos (ordenada $v(20)$ ). No realiza cálculo numérico alguno.

E. $-¿$ Crees tú que ésa es la distancia que recorre en los 20 segundos?

C84 -No. Ésa es la del segundo 20.

E. -Bien, pero te pido la distancia que ha recorrido en los 20 segundos, no en el segundo 20.

C84 -Ya. Es ésta. [Va trazando las ordenadas en $t=19$, $t=18, \ldots]$ 
E. -Bien. ¿Cómo calculas la distancia total recorrida?

C84 -Sumando todas esas alturas.

Cada indivisible representa para él una distancia recorrida. La suma de los indivisibles representa, pues, la distancia recorrida. Le asigna 20 indivisibles, que son las ordenadas en $t=1, \ldots, t=20$. Implícitamente, para este estudiante, a una reunión de indivisibles diferentes, corresponde siempre una suma de medidas.

E. $-i$ Has pensado que el coche, entre $t=19$ segundos y $t=$ 20 segundos, por ejemplo, también anda?

C84 -Sí, claro. [Sigue trazando indivisibles.]

E. ¿¿Podrías calcular...?

C84 -¡Hay infinitas alturas! ¿Cómo puede ser?

E. $-i$ Me puedes explicar qué no puede ser?

C84 -Sólo hay 20 segundos, pero entre 0 y 20 hay infinitos puntos...

Al llegar a este punto, se encuentra ante un conflicto, porque parte de la premisa falsa de que el continuo está formado por elementos indivisibles. Aquí los estudiantes pueden mezclar dos tipos de infinito distintos: la divisibilidad infinita del espacio en el intervalo [0,20] con la extensión infinita que se aplica al tiempo.

De repente, ve la luz asociando las alturas con el proceso de integración.

C84 -Ya sé cómo resolverlo.

E. -Díme.

C84 -El espacio que recorre es esta área [Señala bien.], y con estas alturas se halla la altura media... es una integral...

Los estudiantes C74 y C65 comienzan igual que el C84, trazando indivisibles, y se quedan sin respuesta a la hora de resolver el problema.

Para C65, considerar el tiempo como una sucesión de números (magnitud discreta) en vez de un continuo no le va a permitir poder asociar el área con la distancia.

C65 -Calculo el espacio sumando estas distancias. [Señala las ordenadas.]

E. $-i$ Has pensado que el coche, entre $t=19$ segundos y $t=20$ segundos, por ejemplo, también anda?

C65 -iClaro! En 19 segundos anda esto [Señala la ordenada en $t=19$.], y en el segundo 20, esto. [Señala la ordenada en $t=20$.].

El espacio recorrido para él es la suma de las 20 ordenadas. No puede ver un área, ya que quedan «huecos» entre dos ordenadas sucesivas. Al considerar el tiempo como cantidad discreta, no es posible encontrar una frontera común entre sus partes.

A partir de este momento resuelve el problema.

Ningún estudiante hace mención de la unidad de medida al resolver el problema.
Estos razonamientos nos llevan a la siguiente conclusión: Los estudiantes que parten de la premisa falsa de que el continuo está formado por elementos indivisibles tienen dificultades para reconocer y calcular el área bajo la curva velocidad como el espacio recorrido; no así los que recurren a una relación funcional entre las tres variables: $e=v \cdot t$. Es indudable que el acercamiento a la integral como medida del área de un rectángulo congruente con la región curvilínea permite una transferencia inmediata a esta relación, ya que asocian el tiempo con la base y la velocidad con la altura.

Problema 3. (Ver Anexo). Probabilidades geométricas

Presentamos en este problema otra situación nueva para el estudiante. Se trata de un problema de probabilidades geométricas para cuya solución no es posible contar los casos favorables y los posibles, como ocurre en los juegos de azar discretos, sino que se deben medir dichos casos: la medida de los puntos de un dominio convexo es igual a su área.

Este problema ha sido abordado por todos los estudiantes con un solo tipo de respuesta, aunque expresada de muy distinta forma:

a) La probabilidad de dar en la diana es el área bajo la curva dividida por el área del rectángulo.

Este modelo de respuesta corresponde a 7 de los 9 estudiantes entrevistados. Se les facilita la hoja con el enunciado del problema presentado como en los anteriores de forma diagramática, y se les invita a resolverlo.

El estudiante C76 pregunta antes de comenzar:

C76 - ¿La probabilidad es «eso» de casos favorables partido por posibles?

E. -Sí.

C76 -Pues, ya está. Será el área de $O A B$ dividido por la de $O A B C$.

E. $-i$ Me puedes explicar...?

C76 -El dardo tiene que dar en cualquier punto de esta área [Señala el área bajo la curva.], pero puede tener mala puntería y dar en esta parte [Señala la parte no rayada del gráfico.] y aquí no valdría.

Evidentemente que, aunque hay un empleo tácito de que la probabilidad es la misma en toda la parte sombreada, no son conscientes de ese razonamiento. Sólo al estudiante C75, se le plantea momentáneamente una duda:

C75 - ¿Da lo mismo dar en un punto que en otro?

E. -Sí. ¿Por qué lo preguntas?

C75 -Porque en las dianas siempre ganas puntos distintos si das en un trozo rojo o en uno verde, por ejemplo... ; Ya entiendo! Pero da lo mismo dar en cualquier punto del trozo rojo... ya, ya...

b) La probabilidad de dar en la diana es casos favorables dividido por posibles. 
Esta respuesta se puso de manifiesto en dos estudiantes, aunque no de una forma explícita; ninguno fue capaz de resolver el problema debido a no asociar los casos favorables y posibles con la medida de las áreas.

C86 -La probabilidad es que dé aquí [Señala la región sombreada rayada.] dividido por lo que dé aquí [Señala la parte blanca.]

En su contestación deja claro, que en los casos posibles, no los incluye todos. Parece que en este estudiante predomina la idea de que la probabilidad es: casos favorables dividido por los desfavorables. Sólo es capaz de utilizar el lenguaje gestual.

C66 - Los casos favorables es que dé en el sitio bueno y los posibles en cualquier sitio.

E. $-i$ Me puedes señalar en el gráfico a qué te refieres cuando dices «cualquier sitio» $\mathrm{y}$ «sitio bueno»?

C66 -Aquí [Señala el rectángulo.] es todo y aquí [Señala el área bajo la curva.] es donde tiene que dar el dardo.

E. - ¿Me podrías explicar cómo la calculas?

C66 -Los casos favorables dividido por todos.

E. -Muy bien. ¿Cómo los hallas?

C66 -Los favorables, 3, y todos, 4. Será 3/4.

E. -Indícame cómo has llegado a esa solución.

C66 -Favorables son estos 3 puntos [Señala $0, A$ y $B$.] y todos son estos 4 [Señala $0, A, B$ y $C$.]

Vuelve a poner de manifiesto, como ya ocurrió en el caso del cuestionario del infinito, la idea que tiene de región. Para él, los puntos que determinan la región son los vértices, $y$, aunque señala bien, a la hora de decir cuál es la región de los casos favorables y posibles, no puede pensar en la infinitud de sus puntos. Sin embargo, cuando se refiere a un segmento, no le asigna dos puntos, como era de esperar, sino 30 ó 40, como ya manifestó en la primera entrevista.

Concluímos que la transferencia al contexto probabilístico ha sido posible, ya que, al existir en ellos un sustrato natural intuitivo para las nociones de probabilidad, los estudiantes han asociado la medida de los puntos del dominio con su área, evidentemente, sin realizar ningún razonamiento explícito.

\section{REFLEXIÓN FINAL}

La introducción a la integral definida mediante su definición geométrica permite establecer una relación integral-medida que favorece la transferencia a otros contextos. De esta forma se están sentando las bases para que el estudiante se dé cuenta de que allí donde existe una función que relaciona dos magnitudes, de manera que a cada valor de una de ellas corresponde un determinado valor de la otra, existe un problema de cálculo integral. El cálculo integral determina los resultados de los cambios entre esas dos magnitudes. Es muy impor- tante la imagen visual que les permite darse cuenta de que los resultados de los cambios y las áreas bajo los gráficos son exactamente lo mismo desde el punto de vista de las matemáticas.

Una conclusión que parece muy clara en este trabajo es que, en la secuenciación de contenidos, debe primar su génesis histórica-al parecer más en consonancia con las ideas y el proceso de aprendizaje de los estudiantessobre su orden lógico, y que la introducción a los conceptos mediante la resolución de problemas que han estado en el origen del concepto logra realzar la formación de éste. Al utilizar simultáneamente diferentes representaciones, se favorece el establecimiento de conexiones entre ellas, siendo estas conexiones las que marcan las diferentes etapas del aprendizaje en los estudiantes. Aquí es donde el ordenador juega un papel importante debido a su potencia visual, que ayuda a la formación y transformación de intuiciones y a la creación de imágenes del concepto, y debido también a la facilidad para realizar cálculos, eximiendo al estudiante de esta tediosa labor. De esta forma el estudiante puede centrarse en la exploración y discusión de los conceptos. Los errores cometidos por los estudiantes sirven para acrecentar su aprendizaje y completar así sus imágenes del concepto.

\section{NOTAS}

${ }^{1}$ Hemos adoptado esta traducción para significar el término que acuñaron Tall y Vinner (1981) como concept-image para diferenciar los conceptos matemáticos definidos formalmente y los procesos cognitivos utilizados para concebirlos (Vinner, 1991). Para Vinner, concepto es lo que se deriva de su definición matemática, entendiendo pordefinición del concepto una secuencia de palabras o definición verbal que explica el concepto con precisión, e imagen del concepto es lo que se refleja en la mente de cada individuo, pudiendo ser una representación visual, o bien una serie de impresiones o experiencias. Es, por lo tanto, algo no verbal que se asocia en la mente con el nombre del concepto y que se ha ido formando a través de los años por medio de experiencias de todo tipo y que puede que contenga partes que no estén de acuerdo con la definición formal o con otras de la propia imagen. Existen en la literatura de investigación otras palabras para expresar algo parecido, tales como objeto mental, concepción, representación mental, modelo conceptual, esquema conceptual, etc.

${ }^{2}$ Para Lakatos, los errores, las dudas y las contradicciones son otras tantas indicaciones sobre la manera en que se han desarrollado los conocimientos matemáticos en la historia. Tienen, por tanto, un papel fundamental en el crecimiento de una disciplina.

${ }^{3}$ El desarrollo completo de este estudio queda recogido en Turégano (1993a).

${ }^{4}$ Hemos estudiado la evolución del sistema educativo español a lo largo del siglo XX y hemos analizado textos del siglo XIX y del siglo XX. El desarrollo de este estudio se encuentra en Turégano (1996a).

${ }^{5} \mathrm{La}$ integral de Riemann se refiere a funciones acotadas definidas en un intervalo compacto. Si el intervalo o la función no son acotados, es preciso establecer nuevas definiciones. La noción de integral de Lebesgue, en cambio, se refiere a cualquier 
función medible, definida en un conjunto arbitrario y acotada o no en él.

${ }^{6}$ Lebesgue utiliza el término dominio para referirse a los puntos de la región interior que determina en el plano toda curva cerrada sin puntos múltiples.

${ }^{7}$ La existencia, propiedades y teorema fundamental, los encontramos en Turégano (1991, 1992, 1994, 1996a y b).

${ }^{8}$ Véase DCB, pp. 482, 485, 486, 487, 520, 521, 528, 529 y 531.

${ }^{9}$ Utilizamos el término intuición para las formas de conocimiento directas, globales y evidentes, tal como lo hace Fischbein y otros (1979).

\section{REFERENCIAS BIBLIOGRÁFICAS}

AZCÁRATE, C. (1990). La velocidad: introducción al concepto de derivada. Tesis doctoral. Universitat Autónoma de Barcelona.

BORASI, R. (1987).Exploring Mathematics through the Analysis of Errors. For the Learning of Mathematics, 7(3), pp. 2-9.

CORDERO, F. (1987). El cálculo diferencial e integral como un solo concepto: la derivada. Xalapa (Veracruz. México): Memorias del IX Congreso Nacional de la Asociación de Profesores de Matemáticas, pp. 232-239.

DREYFUS, T. (1990). Advanced Mathematical Thinking, en Nesher, P. y Kilpatrick (eds.), Mathematics and cognition, pp. 113-134. Cambridge University Press.

DREYFUS, T. (1991a). Advanced Mathematical Processes. Tall D. (ed.), Advanced Mathematical Thinking, pp. 25-45. Londres: Kluwer Academic Publishers.

DREYFUS, T. (1991b). On the Status of Visual Reasoning in Mathematics and Mathematics Education. Proceedings of the 15th International Conference PME, 1, pp. 33-48. Italia.

DREYFUS, T. y EISENBERG, T. (1990). On Difficulties with Diagrams: Theoretical Issues. Proceedings of the XIVth International Conference PME, 1, pp. 27-34. Oaxtepec: México.

EISENBERG, T. y DREYFUS, T. (1991). On the Reluctance to Visualize in Mathematics, en Zimmermann, W.y Cunningham, S. (eds.), Visualization in Teaching and Learning Mathematics, pp. 25-37. Washington: Mathematical Association of America.

FILLOY, E. (1981). Investigación en Matemática Educativa en México. Recherches en Didactique de Mathématiques, 2 , pp. 233-256.
${ }^{10}$ Entendemos, por situaciones didácticas, «situaciones de enseñanza en las que se intersectan trayectorias que se trazan de distintos campos del saber, como son la psicología, la pedagogía, la epistemología, etc., hacia un problema específico de enseñanza de las matemáticas» (Glaesser, 1981).

${ }^{11}$ El modelo constructivista inspira una nueva forma de enfocar las actividades escolares y se basa en el hecho de que los estudiantes tienen unos conocimientos previos sobre los fenómenos que se estudian, y que es necesario que el profesor conozca y aproveche este punto de partida, provocando en ellos una reestructuración de sus ideas que les permita evolucionar en su comprensión del mundo cotidiano y científico (Glasersfeld, 1987).
FISCHBEIN, E., TIROSH, D. y HESS, P. (1979). The intuition of infinity. Education Studies in Mathematics, 10, pp. 3-40.

FREUDENTHAL, H. (1973). Mathematics as an Educational Task. Dordrecht: Reidel.

GLAESSER, G. (1981). Epistémologie des nombres relatifs. Recherches en Didactique des Mathématiques, 2(3), pp. 303-346.

GLASERSFELD, E. (1987). Learning as a constructive activity, en Problems of representation in the teaching and learning of mathematics. Janvier, C. (ed.). Londres: LEA.

HEID, M.K. (1988). Resequencing skills and concepts in applied calculus using the computer as a tool. Journal for Research in Mathematics Education, 19(1), pp. 3-25.

LAKATOS, I. (1976). Proofs and Refutations. The Logic of Mathematical Discovery. Cambridge: Cambridge University Press.

LEBESGUE, H. (1928). Leçons sur l'intégration et la recherche de fonctions primitives. París: Éditions Jacques Gabay.

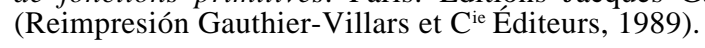

LEBESGUE, H. (1931). Measure and the Integral. Berkeley, (California): Holden-Day. (Reimpresión, 1966).

MEC (1989). Diseño curricular base. Madrid: MEC.

ORTON, A. (1979) An investigation into the understanding of elementary calculus in adolescents and young adults. Cognitive Development Research in Science and Mathematics, pp. 201-215.

ORTON, A. (1983a). Students' understanding of integration. Educational Studies in Mathematics, 14, pp. 1-18. 
ORTON, A. (1983b). Students' understanding of differentiation. Educational Studies in Mathematics, 14, pp. 235-250.

SCHNEIDER-GILOT, M. (1988). Des objets mentaux «aire» et «volume» au calcul des primitives. Tesis doctoral. Université Catholique de Louvain. Faculté des Sciences. Louvain La Neuve.

SIERPINSKA, A. (1985a). La notion d'obstacle épistémologique dans l'enseignement des Mathématiques. Proceedings of the 37th CIEAEM's Meeting, pp. 73-93. Leiden.

SIERPINSKA, A. (1985b). Obstacles épistémologiques relatifs à la notion de limite. Recherches en Didactique de Mathématiques, 6(1), pp. 5-67.

SIERPINSKA, A. (1987a) Humanities students and epistemological obstacles related to limits. Educational Studies in Mathematics, 18(4), pp. 371-397.

SIERPINSKA, A. (1987b). Trying to overcome epistemological obstacles relative to limits in 17 year old humanities students. Proceedings of the 38th CIEAEM's Meeting, pp. 183-193. Southampton.

SIERPINSKA, A. (1989). How \& when attitudes towards mathematics \& infinity become constituted into obstacles in students. Proceedings of the 13th International Conference PME, pp. 166-173.

TALL, D. (1985). Understanding the calculus. Mathematics Teaching, 110, pp. 49-53.

TALL, D. (1986). A graphical approach to integration. Mathematics Teaching, 114, pp. 48-51.

TALL, D. (1991). Intuition and rigour: The role of visualization in the calculus, en Zimmermann, W. y Cunningham, S. (eds.), Visualization in Teaching and Learning Mathematics, pp. 105-119. Washington: Mathematical Association of America.

TALL, D. y VINNER, S. (1981). Concept image and concept definition in mathematics, with particular reference to limits and continuity. Educational Studies in Mathematics, 12, pp. 151-169.

THOMAS, M.O.J. (1988). A conceptual approach to the early learning of algebra using a computer. Tesis doctoral sin publicar. Universidad de Warwick, Inglaterra.
TURÉGANO, P. (1991). Propuesta didáctica para la integral definida. Comunicación presentada en las IV Jornadas de Aprendizaje y Enseñanza de las Matemáticas. Castellón, 2023 de mayo de 1991.

TURÉGANO, P. (1992). Una alternativa a la integral de Riemann, Ensayos, 6, pp. 227-233.

TURÉGANO, P. (1993a). De la noción de área a su definición. Investigación histórica sobre las técnicas, métodos y conceptos que condujeron a la teoría de la medida. Cuenca: Servicio de Publicaciones de la Universidad de Castilla-La Mancha.

TURÉGANO,P. (1993b). Estrategias que utilizan los estudiantes en la resolución de problemas de áreas. Comunicación presentada en el IV Congreso Internacional de Investigación en la Didáctica de las Ciencias y las Matemáticas. Enseñanza de las Ciencias, Vol. extra, pp. 353-355.

TURÉGANO, P. (1994). Los conceptos en torno a la medida y el aprendizaje del cálculo infinitesimal. Tesis doctoral en microfitxes. Servei de Publicacions. Universitat de València.

TURÉGANO, P. (1995). El currículo y las dificultades de aprendizaje del cálculo infinitesimal. Ensayos, 10, pp. 217-233.

TURÉGANO,P.(1996a).Área e integral. Investigación histórica acerca de la transmisión de los conceptos de área $e$ integral. Albacete: Servicio de Publicaciones de la Universidad de Castilla-La Mancha.

TURÉGANO, P. (1996b). Reflexiones didácticas acerca del concepto de área y su medida, Uno, 10, pp. 9-27.

TURÉGANO, P. (1996c). Intuición del infinito en estudiantes de primero de BUP. Épsilon, 34, pp. 11-46.

VINNER, S. (1989). Avoidance of visual considerations in calculus students. Focus: on learning problems in mathematics. Visualization and Mathematics Education, 11, 2, pp. 149-155.

VINNER, S. (1991). The role of definitions in the teaching and learning of mathematics, en Advanced Mathematical Thinking, pp. 65-81. David Tall (ed.). Londres: Kluwer Academic Publishers.

[Artículo recibido en febrero de 1996 y aceptado en noviembre de 1997.] 


\section{ANEXO}

PROBLEMAS PLANTEADOS EN LA ENTREVISTA POSTAPRENDIZAJE

Problema 1. El diagrama muestra el gráfico de $f(x)=x^{3}-2 x^{2}$. Calcula el área bajo la curva, desde $x=0$ hasta $x=3$.

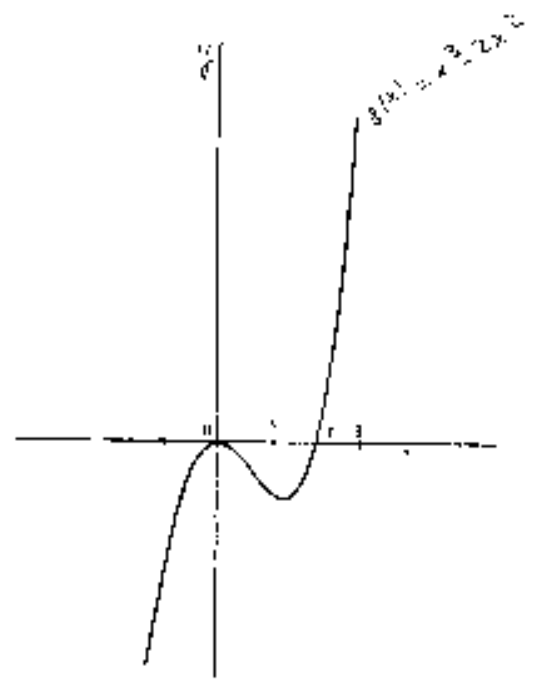

Problema 2. Un coche de fórmula 1 se queda sin propulsión debido a una rotura de embrague. Se estima que a partir de ese momento $(t=0)$ su velocidad en metros por segundo vendrá dada por $v=80-0$ '05 $t^{2}$, cuya gráfica puedes comprobar que es la de la figura adjunta.

- ¿Qué distancia habrá recorrido a los 20 segundos?

- ¿Qué distancia recorre entre los 20 y 40 segundos?

- ¿Qué distancia recorrerá hasta pararse?

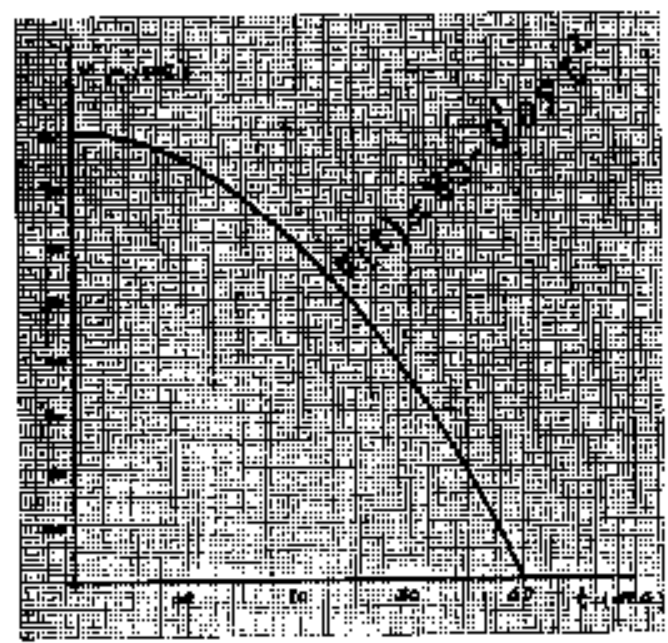


Problema 3. Un profesor de matemáticas muy aficionado a tirar dardos en una diana propone a sus alumnos el siguiente problema: Aprovechando parte del gráfico de la función $f(x)=x^{3}$, dibuja una diana OABC.

¿Podrías hallar la probabilidad de que, al tirar un dardo, éste caiga dentro de la parte sombreada de OAB?

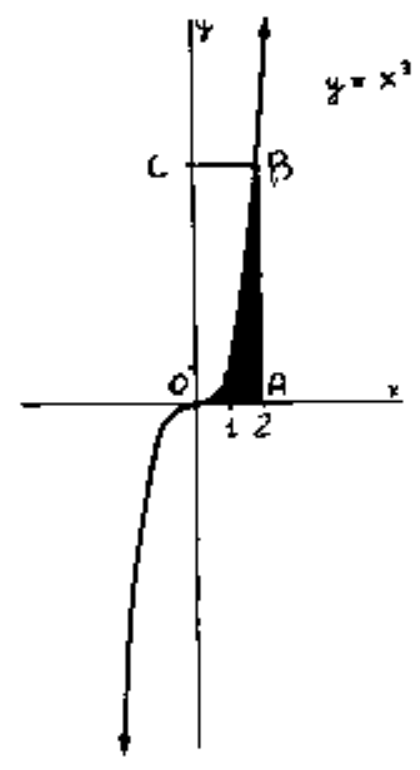

